\title{
Commonplace
}

\section{Annotation as social construction of knowledge}

\section{Paulo Pinto}

Published on: Jul 22, 2021

DOI: $10.21428 / 6 f f d 8432.2751 b 969$

License: Creative Commons Attribution 4.0 International License (CC-BY 4.0). 
Text and writing is deeply intertwined with the world. Indeed, the famous French philosopher Jacques Derrida writes, "il n'y a pas de hors-texte," or "there is no outsidetext," in Of Grammatology. Here, he's claiming that we use context to make sense of things. Text exists in a certain field of meaning and expresses an idea, a thought, a concept, at a given chronological moment. Thus, the text is pure expression of a certain ontological construction, in that its being and meaning is created within the context in which it exists. That context inherently requires a reader of the text, and their own experiences. As such, the text is a production open to reflection, a discourse that opens itself to interpretation, dissent, pure dialogue, and also art of memory. For example, when a reader goes back to a book they had formerly read, they come to it with new memories and new ideas, so the experience of this second read is different.

To interact and dialogue with text, one must use annotation. The act of annotating stimulates thinking, produces meaning and turns a text into a source of unlimited semiotics, the processes and study of making signs; perhaps you star an important quote, or place an $\mathrm{X}$ where you disagree with the text. Annotation can also be source of doxography, where readers become authors by transcribing and commenting on other's ideas. Here we could think of Plato acting as a doxographer for Socrates.

On a more personal level, annotation allows you to revisit opinions by going back and reading your own impressions and words inline the text. Thus, writing down can be used as a time machine of sorts, allowing us to revisit what we once thought about a given subject.

\section{Visit the web version of this article to view interactive content.}

Annotation also fosters serendipity because it creates new paths for us and for others. By writing in the margins, either by hand or digitally, you're creating a dialogue with the text and engaging with it, asking it questions, and giving it counter-arguments. Perhaps this is not unlike the Socratic dialogues, where talking and thinking through ideas "aloud", provides the grounds for new ideas. Through this process, you consolidate an idea in writing. And, through writing, it allows solidifying concepts in long-term memory and increasing understanding. 


\section{Visit the web version of this article to view interactive content.}

An instance of how annotation goes beyond individual understanding and proves to be fundamental for shared and public modes of knowledge is the recent publication of the MIT Press Essential Knowledge Series, Annotation, by Remi H. Kalir and Antero Garcia. This book describes the relationship between reading and writing across historical and contemporary contexts. Indeed, it expands the term from the written marginalia themselves to the process or genre of communicating, thus giving the act of annotating to express power and opinions.

To democratize annotations and sharing the ideas and comments of readers, the authors encourage readers to share notes on Twitter, using the hashtag \#AnnoConvo. $\underline{1}$ This is a book that dialogues with its readers and encourages them to publicly share their notes, ideas, refutations, disagreements.

\section{Visit the web version of this article to view interactive content.}

Based on these open conversations about the book, annotation can still be and should be an excellent tool for teachers and students, as it encourages thinking, turning students into content producers and not just mere recipients of information. Bridging between information, communication, and knowledge, annotation allows refuting the transmissionist model, or the one-way lecture-style model, of interaction and construction of knowledge. Instead, public annotation induces the collective production of knowledge and ways of disseminating and searching for information and allows for effective communication to become an ongoing process.

Far from being a mere a tool for the accumulation and circulation of information, computer technologies provide broad support for the mediation of interactions. For example as a technology, the social software "Hypothes.is" facilitates online registration, organization and retrieval of information, promotes communication and the social construction of knowledge. Even here using the PubPub platform, you can add in-line comments to this text, and others. 
Human knowledge occurs in interaction. Beyond platform specific technologies, scientific, educational and business context, the development of knowledge (both individually and collectively) largely relies on relationships curated and strengthened through dialogue and conversation. To annotate is, thereby, to promote a culture of conversation that is necessary for our development as human beings, as beings endowed with understanding.

\section{Footnotes}

1. It's worth noting too that, in the spirit and integration of public commentary, the authors put this book up for open review before it was published. $\subseteq$ 